\title{
Luc CAPDEVILA, François ROUQUET, Fabrice VIRGILI et Danièle VOLDMAN (dir.), Hommes et femmes dans la France en guerre (1914-1945), Paris, Payot, 2003, 362 pages.
}

\section{Françoise Thébaud}

\section{OpenEdition}

\section{Journals}

Édition électronique

URL : https://journals.openedition.org/clio/1424

DOI : $10.4000 /$ clio. 1424

ISSN : 1777-5299

Éditeur

Belin

Édition imprimée

Date de publication : 1 novembre 2004

Pagination : 273-275

ISBN : 2-85816-755-9

ISSN : 1252-7017

\section{Référence électronique}

Françoise Thébaud, « Luc CAPDEVILA, François ROUQUET, Fabrice VIRGILI et Danièle VOLDMAN (dir.) Hommes et femmes dans la France en guerre (1914-1945), Paris, Payot, 2003, 362 pages. », Clio. Histoire, femmes et sociétés [En ligne], 20 | 2004, mis en ligne le 06 juin 2005, consulté le 24 avril 2022. URL http://journals.openedition.org/clio/1424; DOI : https://doi.org/10.4000/clio.1424

Ce document a été généré automatiquement le 24 avril 2022

Tous droits réservés 


\title{
Luc CAPDEVILA, François ROUQUET, Fabrice VIRGILI et Danièle VOLDMAN (dir.), Hommes et femmes dans la France en guerre (1914-1945), Paris, Payot, 2003, 362 pages.
}

\author{
Françoise Thébaud
}

1 Issu d'un séminaire sur «Identités de genre et guerres au XXe siècle » tenu à l'Institut d'histoire du temps présent pendant trois ans et rédigé par quatre spécialistes de la Deuxième Guerre mondiale, Hommes et femmes dans la France en guerre est une synthèse stimulante de nombreux travaux anciens et récents ${ }^{1}$. C'est aussi un ouvrage important à deux titres au moins. D'une part, il met en oeuvre une double comparaison qui permet d'aiguiser le regard sur les rapports entre guerre et changement social, et notamment sur la question récurrente depuis plusieurs décennies de sa fonction émancipatrice pour les femmes : comparaison entre le premier et le second conflit mondial qui ont pris en France des formes très différentes ; comparaison systématique entre les hommes et les femmes, qui évite la dissymétrie reprochée aux premiers pas de l'histoire des femmes et souligne que cette approche a bien impulsé une histoire des masculinités. D'autre part, il pose de façon neuve les identités (singulières et collectives) comme des objets historiques à part entière, puisque construites socialement et évoluant dans le temps et dans l'espace. Ce faisant, il promeut une histoire du genre et en souligne l'intérêt.

Que veut dire se sentir homme ou femme et comment approcher historiquement les identités de genre ? Ce livre s'ouvre par un utile chapitre méthodologique qui propose définition et clefs de lecture. Perception de soi profondément intériorisée et moteur des comportements, l'identité de genre serait « le produit d'un perpétuel ajustement entre les situations (réelles), les rôles (imaginaires) et les places (symboliques) de l'un et l'autre sexe » (p. 31). Par définition instable, il est particulièrement intéressant de 
l'observer en période de guerre qui met «des individus et des groupes face à euxmêmes et aux autres dans des épreuves de vérité » (p. 39).

3 L'impact des deux conflits mondiaux «a-t-il favorisé la transformation des identités masculines et féminines dans le courant du XXe siècle? » (p. 11). Les auteurs tentent d'y répondre en trois parties. Intitulée "Mobilisations ", la première, concrète et chiffrée, rappelle les formes sexuées de mobilisation pendant la Grande Guerre et souligne l'évolution, d'un conflit à l'autre, de la place des femmes dans les dispositifs de la nation en armes. Inscrite dans la loi, longtemps débattue, du 11 juillet 1938 sur "l'organisation générale de la nation pour le temps de guerre » qui prévoit un cadre réglementaire de recrutement des civils incluant les femmes et dans les décrets du printemps 1940 qui fondent le statut des «auxiliaires féminines des formations militaires ", cette évolution n'est pas stoppée par l'armistice et le familialisme de Vichy: de part et d'autre, les discours de mobilisation incluent les femmes qui sont présentes dans la collaboration, la résistance intérieure, les armées de la France libre ou partent travailler en Allemagne (70000). L'investissement du sexe par la puissance publique en guerre est l'objet des trois chapitres suivants qui déclinent pour les deux conflits les formes prises par la politisation des corps et les mesures adoptés par un Etat qui tente, avec plus ou moins de succès, de se faire chaperon, père, démographe, gendarme et proxénète. Mais c'est la troisième partie qui répond le mieux, au risque de répétitions de faits et d'anecdotes déjà cités, à la question initiale. Elle observe en effet ce que deviennent les assignations de genre à l'épreuve du feu, soulignant «une tendance à l'indifférenciation des attributs féminins et masculins " (par exemple la communauté de souffrance sous les bombes), voire des phénomènes d'inversion des rôles : faiblesse des hommes traumatisés, invalides, vaincus ou prisonniers ; puissance des femmes chefs de famille, salariées, infirmières, résistantes ou engagées dans l'armée. À cet égard, les pages les plus neuves évoquent la souffrance des hommes au combat, leurs tentatives pour y échapper, leur peur de perdre toute virilité. Les auteurs insistent de nouveau sur la révolution mentale qu'entraîne, à partir de la Deuxième guerre, la présence de femmes dans l'armée (voire au combat), femmes désormais prises en compte dans le droit de la guerre. Mais ils rappellent aussi l'un des dix commandements des Merlinettes de la France libre : «Toute ta vie, femme soldat / Ta féminité garderas ». Crainte, exprimée par les hommes ou les femmes, du brouillage des genres? Tentative de conjurer la nouveauté ? Stratégie pour se faire accepter ? Sur cet aspect comme sur d'autres, l'interprétation n'est pas univoque et peut conduire le lecteur à s'interroger sur la démonstration générale de l'ouvrage.

4 Trois points en effet font débat ou méritent discussion. Tout d'abord, un point de chronologie : la volonté de comparer les deux guerres conduit les auteurs à considérer comme "un tout» la période 1914-1945, à entériner la notion problématique d'un « entre-deux-guerres » et à le considérer de façon réductrice comme un moment « où la société a essayé de retrouver la paix en anticipant le conflit à venir» (p. 9) ; de même, l'usage des décennies 1920 et 1930 pour souligner telle ou telle évolution peut paraître trop facile. D'autre part, un point de vocabulaire : si l'approche historique des identités et le choix des mots pour le dire ne sont pas faciles, l'usage de termes nuancés reste de rigueur, comme "vacillement», «brouillage», éventuellement "croisements identitaires »; mais, plusieurs fois répétée, «l'errance identitaire » peut sembler une inutile dramatisation, peu compatible avec la notion "d'ajustement» tant de fois utilisée. Enfin, l'ouvrage défend, contre des travaux précédents parfois caricaturés ${ }^{2}$, la thèse d'un « changement en profondeur » des relations entre les hommes et les femmes 
par la guerre : rapprochement des rôles et atténuation de la hiérarchie. Mais l'analyse de détail utilise de façon récurrente conjonctions et adverbes qui introduisent de fortes nuances à cette affirmation, présente des interprétations aux tonalités différentes d'une partie à l'autre (par exemple à propos des femmes dans l'armée) ou conclut de façon prudente, soulignant la complexité des phénomènes : «les deux guerres, est-il écrit en conclusion, se sont donc révélées des moments uniques, sortes de points nodaux de l'évolution des identités de genre qu'elles ont précipitée, même si cette accélération n'augurait pas de changements définitifs ou d'avancées pérennes» (p. 290). L'évolution des identités, sans doute très inégalement partagée, n’a pas nécessairement, dans des contextes réactifs d'après-guerre, des effets immédiats ou sans concurrence sur les rôles sociaux... Reste un livre foisonnant qui invite à creuser le sillon des identités et apprend beaucoup sur le XXe siècle.

\section{NOTES}

1. Parmi les travaux récents, citons : Jean-Yves Le Naour, Misères et tourments de la chair durant la Grande Guerre. Les moeurs sexuelles des Français, 1914-1918, Paris, Aubier, 2002, 411 pages.

2. L'auteure de ce compte rendu a certes défendu, en réaction aussi contre une historiographie précédente très optimiste, l'idée d'une guerre plus conservatrice qu'émancipatrice. Mais elle l'a fait avec beaucoup de nuances comme le montre le titre original (conservé dans la version italienne) du chapitre de l'Histoire des femmes en Occident : « La Grande Guerre : "l'âge de la femme" ou le triomphe de la division sexuelle?». 